\title{
Enseñanza en investigación en urología. Análisis bibliométrico
}

\section{Teaching in Urology Research. Bibliometric Analysis}

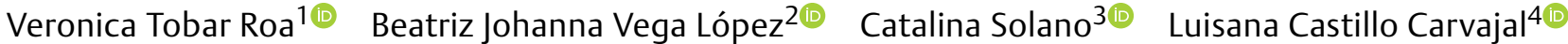 \\ Joan Stefany Duque Pulido ${ }^{5}$ Catalina Gonzalez Yepes ${ }^{3 \odot}$ Pamela Perdomo Barbosa6 ${ }^{6}$
}

1 Uróloga, Centro Urológico Foscal, Bucaramanga, Colombia

2 Uróloga, Magister en Epidemiología, Hospital Universitario de La Samaritana, Bogotá, Colombia

3 Uróloga, Uroclin, Medellín, Colombia

${ }^{4}$ Uróloga, Clínica Juan N corpas Bogotá, Colombia

${ }^{5}$ Uróloga, Hospital infantil universitario de San José, Bogotá, Colombia

6 Uróloga, Clinica Antioquia, Medellín, Colombia

Urol Colomb 2020;29:195-201.
Address for correspondence Beatriz Johanna Vega López, MD, MSc, Hospital Universitario de La Samaritana, Dirección: Calle 140 \# 13-66, apartamento 1401, torre 3, Bogotá 110121, D.C., Colombia (e-mail: drajohannavL@gmail.com).

\section{Resumen}

\section{Palabras clave}

- educación

- investigación

- urología

- publicaciones

- bibliométrico

- medicina en la literatura
Objetivo Caracterizar el panorama de la educación en investigación en urología mediante un análisis bibliométrico.

Métodos Realizamos un análisis bibliométrico, utilizando artículos publicados entre 1955- 2019, sin restricción en el idioma. Se utilizaron las herramientass estadísticass PubMed, FABUMED y PubReminer para la obtención de la información de las variables y realización del análisis bibliométrico. Analisis de mapeo utilizando el software: VOSviewer,. Para la obtención del factor de impacto (FI), se utilizó el Journal Citation Reports 2017/2018.

Resultados Desde 1955 hasta 2019 se publicaron 718 referencias en 245 revistas. A partir del 2000 encontramos un aumento significativo en el número de publicaciones con un punto de corte en el año 2009. Las revistas con mayores publicaciones fueron: ] Urol (8.6\%), BJU Int (6.8\%) y Urology (6.5\%). De las 20 revistas con mayor número de publicaciones, sólo desolo 5 un Fl mayor a 3. Los paises con mayor publicacion fueron: Estados Unidos (23,6\%), Reino Unido (20,2\%). El país de América Latina con mayor publicacion fué Brasil $(0,8 \%)$.

Conclusiones El estado de la educación en investigación en urología demuestra un crecimiento en la producción científica, con una baja contribución por parte de los países latinoamericanos. Para poder desarrollar una creación intelectual de calidad se deberá invertir tiempo y recursos en un adecuado entrenamiento en investigación en los programas de formación en urología.

Objective Characterize the panorama of urology research education through a bibliometric analysis.

Methods We performed a bibliometric analisys, using articles published between 1955-2019, without restriction on language. The statistical tools PubMed, FABUMED
DOI https://doi.org/ 10.1055/s-0040-1721330. ISSN $0120-789 \mathrm{X}$ e ISSN 2027-0119.
Copyright (c) 2020, Thieme Revinter Publicações Ltda.., Rua do Matoso 170, Rio de Janeiro, RJ, CEP 20270-135, Brazil. Todos los derechos reservados.
License terms

c) $(\oplus) \$$ 


\section{Keywords}

- education

- research

- urology

- publications

- bibliometric

- medicine in literature and PubReminer were used to obtain the information on the variables and perform the bibliometric analysis. We performed a bibliometric mapping analysis using the software program: VOSviewer. To obtain the impact factor (FI), the Journal Citation Reports 2017/2018 was used.

Results From 1955 to 2019, 718 references were published in 245 journals. Starting in 2000 , we found a significant increase in the number of publications with a cut-off point in 2009. The journals with the largest publications were: J Urol (with 8.6\%), BJU Int (with $6.8 \%$ ) and, Urology (with 6.5\%). Of the 20 journals with the highest number of publications, only of the 20,5 had an IF greater than 3 . The countries with the highest publication were: United States (23.6\%), United Kingdom (20.2\%). The Latin American country with the highest publication was Brazil with (0.8\%).

Conclusions The state of education in urology research shows a growth in scientific production with a high impact factor, with a decrease without finding a significant contribution from Latin American countries. In order to develop a quality intellectual creation, time and resources should be invested in adequate research training in urology training programs.

\section{Introducción}

La investigación médica genera los conocimientos necesarios para garantizar una atención en salud costo efectiva y de calidad, de la mano de los avances científicos que se producen día a día. Por tal razón, la educación médica ha visto una transformación trascendental en los contenidos y la forma en cómo se enseña investigación, tanto en programas de pregrado como de postgrado. ${ }^{1}$

En las especialidades médico-quirúrgicas existe un creciente interés en actualizar la estructura de los planes de estudio mediante la capacitación en medicina basada en la evidencia (MBE), así como la producción de trabajos de investigación y la publicación de los mismos. ${ }^{2}$

Los programas de postgrado en urología no han estado exentos de la implementación de la práctica clínica basada en la evidencia (PCBE) y de la enseñanza de metodología de la investigación. ${ }^{3,4}$ Sin embargo, las encuestas realizadas a urólogos graduados y residentes demuestran que, con la educación actual, no se logran adquirir las competencias necesarias en investigación y esto genera una barrera importante para su práctica diaria. ${ }^{2,4-6}$

Entendiendo entonces la importancia de reconocer el estado actual del entrenamiento en investigación en nuestra especialidad, el presente trabajo pretende evaluar las investigaciones relacionadas a la educación en investigación en urología durante los últimos 65 años mediante un análisis bibliométrico, proporcionando a los urólogos una mejor comprensión de las tendencias de publicación acerca de este tema, así como el planeamiento de estrategias educativas en los diferentes programas de residencia.

\section{Métodos}

Se realizó un análisis bibliométrico descriptivo y retrospectivo de la literatura médica obtenida en MEDLINE en febrero 1-
2020, a través de PubMed , utilizando la estrategia de búsqueda con términos (education research urology[Title/Abstract]) incluyendo todos artículos publicados entre 1955 y 2019, sin restricción en el idioma.

Se utilizaron las herramientas estadísticas de FABUMED y PubReminer para la obtención de la información de las variables necesarias para el análisis, entre ellas: número total de publicaciones y revistas; número y porcentaje de publicaciones por revista y país; y patrón de producción científica a lo largo del período estudiado. Adicionalmente realizamos un análisis de mapeo bibliométrico utilizando el software VOSviewer que explora las tendencias de palabras clave y los conceptos relacionados, así como mapas de redes de colaboración.

Se incluyeron artículos originales de investigación, artículos de revista, ensayos, informes científicos y reseñas, con el fin de analizar toda la literatura médica indexada en relación con el tema en estudio.

Para la obtención del factor de impacto (FI), se usó el Journal Citation Reports 2017/2018 de las revistas encontradas para estimar así la calidad de cada una de estas. Para la obtencion de $\mathrm{H}$-index de los autores se utilizó el buscador Scopus.

Posteriormente, se realizó el análisis de los resultados de forma estadística y descriptiva y con base en esta información, se elaboraron tablas y gráficas en Microsoft Office Excel 2017.

\section{Resultados}

\section{Datos generales}

Desde 1955 hasta 2019 se publicaron un total de 718 referencias en 245 revistas. Se graficó de manera independiente de acuerdo a los sucesos que marcaron el número de publicaciones en investigación. En el periodo comprendido entre 1955 y 1999 permaneció estable el 
600

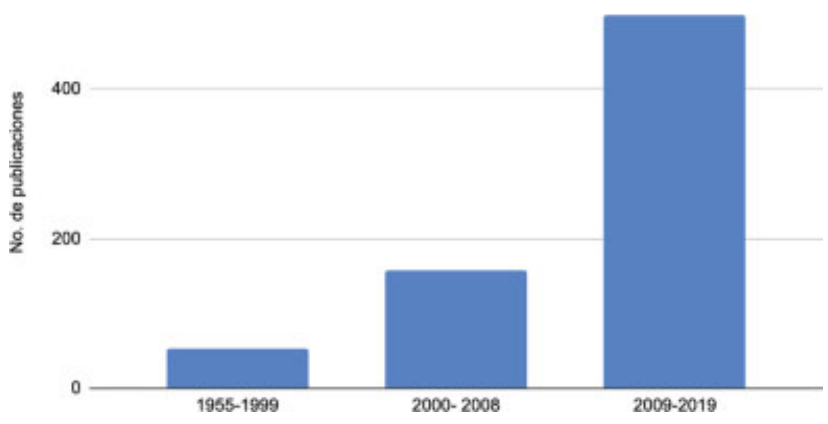

Fig. 1 Crecimiento del número de publicaciones desde 1955-2019 divididas por periodos.

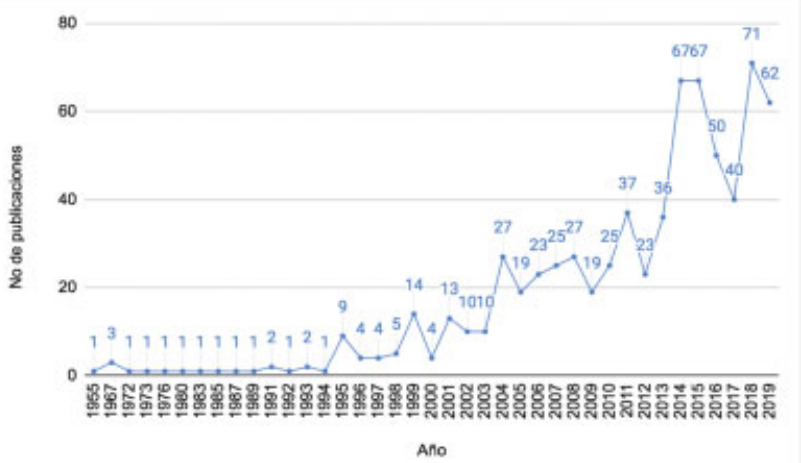

Fig. 2 Crecimiento de publicaciones globales en las últimas 6 décadas acerca de educación en investigación en urología.

número de publicaciones, siendo la producción porcentual el 7.5\% (54/718). A partir del 2000 encontramos un aumento del número de las mismas, con un punto de corte en el año 2009, pasando de un incremento porcentual del 22\% (158/ 718) a 69.4\% (498/718) - Figura 1, -Figura 2.

\section{Revista}

El 51\% (362) de los artículos publicados entre 1955 y 2019 se encuentra en las veinte revistas mostradas en la - Tabla 1, así como el número de artículos publicados en cada una de estas y el FI para el año 2017 - 2018 según el Journal Citation Reports. Las revistas con mayor porcentaje de publicaciones fueron: J Urol con 8.6\% (61), BJU Int con 6.8\% (48), Urology con $6.5 \%$ (46), Urologe A con 4.2\% (30), J Endourol con 3.7\% (26) y 3.7\% (16) en Arch Esp Urol, Eur Urol, J Surg, cada una. Solamente cinco de las 20 revistas con mayor número de publicaciones, tuvieron un FI mayor a 3.

\section{Autores}

Se encontraron 3336 autores, de los cuales 612 son autores principales. Los primeros diez autores con sus publicaciones, número de citaciones, h-index e instituciones, se exponen en la - Tabla 2. El autor con mayor número de publicaciones fue Dasgupta P (King's College London y MRC Centre for Transplantation and Urology Centre, London, UK) con 46 artículos y un h-index de 56 .

\section{Artículos más citados}

Dentro de los artículos con mayor número de citaciones, encontramos que la enseñanza de la medicina basada en la evidencia es el tópico más nombrado. El documento que ocupa el primer puesto, con 89 citaciones, es: ¿What Are the Barriers to Residents' Practicing Evidence-Based Medicine? A Systematic Review, de los autores van Dijk, N; Hooft, L; Wieringa-de Waard, M., publicado en 2004. Sólo uno de los artículos es específico para urología y se encuentra publicado en una revista urológica, con un total de 56 citaciones ( - Tabla 3 ).

\section{Países:}

La producción de publicaciones se originó en 59 países (-Figura 3). De éstos, los diez primeros produjeron 625 artículos, siendo responsables del $88 \%$ del total de publicaciones. Los 3 países con mayor número de artículos son Estados Unidos con 23,6\% (187), Reino Unido con $20,2 \%$ (160) y Turquía con 7,4\% (59). El país de América Latina con mayor número de publicaciones fue Brasil con 6 (0,8\%), seguido de México con 3 (0,4\%). Argentina, Bolivia, Colombia, Guatemala, Perú, Uruguay y Venezuela reportan un artículo cada uno.

\section{Instituciones}

Las instituciones con mayor participación en las publicaciones son en su mayoría de Estados Unidos. La Universidad de Michigan - Ann Arbor, ocupa el primer puesto, con 45 artículos sobre el tema. -Figura 4.

\section{Análisis de co-ocurrencia utilizando palabras claves} Mediante VOSviewer se analizaron los 718 artículos, identificando las áreas de investigación más importantes en el tema. Se evaluó el número de co-ocurrencia entre palabras clave y palabras clave por título y resumen. El mapeo de co-ocurrencia realizado con la unidad de análisis de las palabras clave con un mínimo de 5 repeticiones, quedó organizado en ocho grupos (clusters), con un total de 366 términos. (-Figura 5a).

Vemos cómo en el mapeo y análisis de palabras clave sobre educación en la investigación, en el Grupo 1 (rojo) predomina la palabra "humanos". El grupo 2 (verde) se basa en la educación a los residentes y en el grupo 3 (azul) predomina la educación médica continuada, las publicaciones y la enseñanza en investigación clínica.

El análisis y mapeo de la tendencia en línea de tiempo permite identificar la evolución de la literatura sobre el tema estudiado, las palabras clave que se han venido integrando son: análisis costo-beneficio, medicina basada en la evidencia y práctica clínica.

\section{Análisis de co-ocurrencia entre palabras clave, título y resumen}

En el análisis de co-ocurrencia entre palabras clave de títuloresumen, se encuentra un total de 701 palabras clave organizadas en seis grupos, nombradas un mínimo de cinco veces. La - Figura 6 muestra las palabras clave incluidas en el área de investigación, viendo cómo en los últimos tiempos se han incorporado temas como medición 
Tabla 1 Top 20 de revistas con publicaciones sobre educación en investigación en urología durante el período de 1955 a 2019

\begin{tabular}{|c|c|c|c|c|}
\hline Revista & Artículos & FI 2017-2018 & Idioma & País \\
\hline J Urol & 61 & 5.6 & Inglés & Estados Unidos \\
\hline BJU Int & 48 & 4.5 & Inglés & Inglaterra \\
\hline Urology & 46 & 1.8 & Inglés & Estados Unidos \\
\hline Urologe A & 30 & * & Alemán & Alemania \\
\hline J Endourol & 26 & 2.2 & Inglés & Estados Unidos \\
\hline Arch Esp Urol & 16 & 0.3 & Muti-lenguaje & España \\
\hline Eur Urol & 16 & 17.2 & Inglés & Países Bajos \\
\hline J Surg Educ & 16 & 2.2 & Inglés & Estados Unidos \\
\hline J Pediatr Urol & 15 & 1.7 & Inglés & Inglaterra \\
\hline World J Urol & 11 & 2.7 & Inglés & Estados Unidos \\
\hline Am J Surg & 10 & 2.2 & Inglés & Estados Unidos \\
\hline Urol Nurs & 9 & * & Inglés & Inglaterra \\
\hline J Sex Med & 8 & 3.6 & Inglés & Inglaterra \\
\hline Surg Endosc & 8 & 3.2 & Inglés & Estados Unidos \\
\hline Can J Urol & 7 & 1.03 & Inglés & Canadá \\
\hline Can Urol Assoc J & 7 & 1.25 & Inglés & Canadá \\
\hline J Am Coll Surg & 7 & 4.4 & Inglés & Estados Unidos \\
\hline J Clin Nurs & 7 & 1.75 & Inglés & Inglaterra \\
\hline Indian J Urol & 6 & * & Inglés & India \\
\hline J Wound Ostomy Continence Nurses & 6 & 1.62 & Inglés & Estados Unidos \\
\hline
\end{tabular}

( ${ }^{*}$ no hay reporte de Fl en Journal Citation Reports para 2017-2018).

de los resultados en investigación y productividad en publicaciones.

\section{Discusión}

La investigación es una herramienta de desarrollo que se utiliza en todas las especialidades médicas, cuyo crecimiento y aporte está ligado a los avances tecnológicos. ${ }^{7,8}$ Además, es un marcador de calidad de la educación médica y de la práctica clínica. ${ }^{9}$
Los análisis bibliométricos son una herramienta para evaluar el comportamiento de la producción científica en un periodo de tiempo, así como su impacto. ${ }^{8-10}$ En este estudio encontramos un incremento mayor al 60\% en la generación de contenido académico en investigación en urología, con un crecimiento exponencial durante la última década, lo cual es un reflejo claro de la importancia que ha tomado la educación en investigación en los últimos años.

En nuestro trabajo podemos observar que el total de las publicaciones fueron llevadas a cabo en 59 países. La mayor

Tabla 2 Los diez autores con mayor número de publicaciones en investigación en urología desde 1955 hasta 2019

\begin{tabular}{|l|l|l|l|}
\hline Autor & Artículos & Hindex & Institución y País \\
\hline Dasgupta P & 41 & 56 & Guy's Hospital, London, Reino Unido \\
\hline Ahmed K & 38 & 40 & King's College London, London, Reino Unido \\
\hline Khan MS & 27 & 36 & King's College London, London, Reino Unido \\
\hline Kerfoot BP & 19 & 24 & VA Medical Center, Estados Unidos \\
\hline Challacombe B & 17 & 35 & Guy's and St Thomas' NHS Foundation Trust, Londres, Reino Unido \\
\hline Van Cleynenbreugel B & 10 & 20 & Department of Urology, University Hospitals Leuven, Leuven, Bélgica \\
\hline Veneziano D & 10 & 14 & $\begin{array}{l}\text { The Ohio State University Comprehensive Cancer Center, } \\
\text { Columbus, Estados Unidos }\end{array}$ \\
\hline Wagner C & & 33 & Vrije Universiteit Amsterdam, Amsterdam, Países Bajos \\
\hline Aydin A & 10 & 15 & King's College London, London, Reino Unido \\
\hline Mottrie A & 9 & 41 & Onze-Lieve-Vrouw Ziekenhuis, Asse, Bélgica \\
\hline
\end{tabular}


Tabla 3 Los cinco artículos con mayor número de citaciones con respecto a educación en investigación durante 1955-2019:

\begin{tabular}{|c|l|l|l|l|l|}
\hline & Título & Autores & Citaciones & Revista & Año \\
\hline 1 & $\begin{array}{l}\text { What Are the Barriers to Residents' } \\
\text { Practicing Evidence-Based Medi- } \\
\text { cine? A Systematic Review }\end{array}$ & $\begin{array}{l}\text { van Dijk, N; Hooft, L; } \\
\text { Wieringa-de Waard, M. }\end{array}$ & 89 & Academic Medicine & 2010 \\
\hline 2 & Teaching evidence based medicine & $\begin{array}{l}\text { Del Mar, C., Glasziou, P., } \\
\text { Mayer, D. }\end{array}$ & 75 & $\begin{array}{l}\text { British Medical } \\
\text { Journal }\end{array}$ & 2004 \\
\hline 3 & $\begin{array}{l}\text { Clinical research and statistical } \\
\text { methods in the urology literature. }\end{array}$ & $\begin{array}{l}\text { Scales, CD; Norris, RD; } \\
\text { Peterson, BL; et ál. }\end{array}$ & 56 & Journal of Urology & 2005 \\
\hline 4 & $\begin{array}{l}\text { Teaching evidence based medicine } \\
\text { to surgery residents-is journal club } \\
\text { the best format? A systematic re- } \\
\text { view of the literature }\end{array}$ & $\begin{array}{l}\text { Ahmadi, N., McKenzie, M.E., } \\
\text { MacLean, A, Mastracci, T., }\end{array}$ & 51 & $\begin{array}{l}\text { Journal of Surgical } \\
\text { education }\end{array}$ & 2012 \\
\hline 5 & $\begin{array}{l}\text { Beyond journal clubs: Moving } \\
\text { toward an integrated evidence- } \\
\text { based medicine curriculum }\end{array}$ & $\begin{array}{l}\text { Hatala, R., Keitz, S.A., } \\
\text { Wilson, M.C., Guyatt, G. }\end{array}$ & 45 & $\begin{array}{l}\text { Journal of General In- } \\
\text { ternal Medicine }\end{array}$ & 2006 \\
\hline
\end{tabular}

parte de la producción científica se realizó en Estados Unidos, Reino Unido y Turquía, mientras que la contribución de los países latinoamericanos fue menor del 1\%; datos también reflejados en el análisis bibliómetrico realizado por Majzoub y cols, que documentó la estadística de las publicaciones en urología. $^{11}$

El bajo aporte de producción científica de los países latinoamericanos, incluido Colombia, puede deberse a

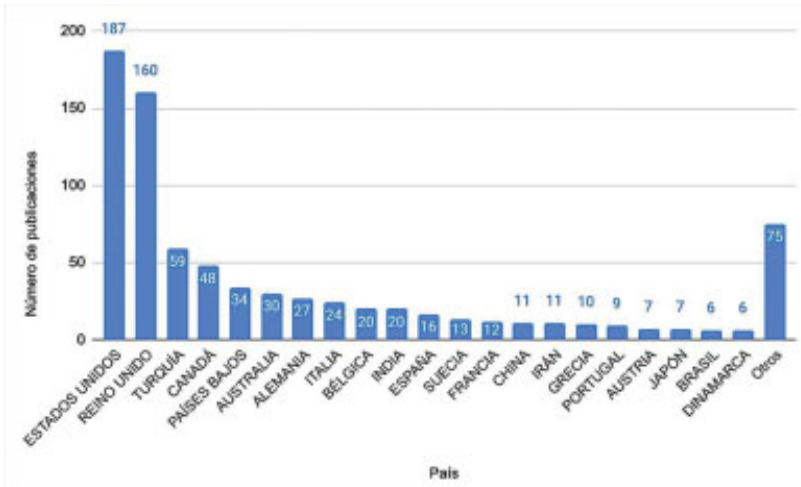

Fig. 3 Países participantes en las publicaciones sobre educación en investigación en urología 1955-2019.

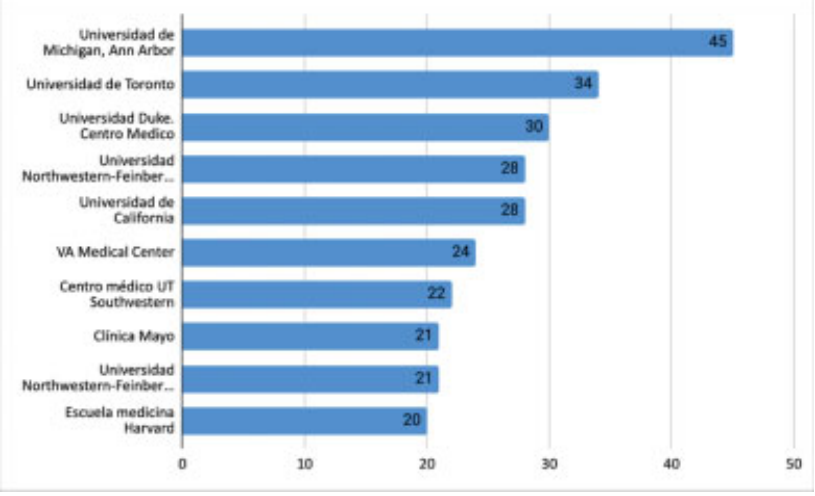

Fig. 4 Top 10 de las instituciones con mayor porcentaje de artículos sobre educación en investigación en urología 1955-2019. varios factores, como la falta de educación formal en investigación en algunos programas de residencia, la ausencia de tiempo protegido para dedicar a la producción intelectual y las dificultades económicas de los países en desarrollo, que se traducen en poco financiamiento para la investigación.

Es claro que hay un aumento en la producción científica y que los programas de formación actual fomentan la medicina basada en la evidencia, como se menciona en el artículo de De Battisti y Salini, ${ }^{3}$ sin embargo, en 2009 Dahn y cols publicaron los resultados de una encuesta realizada en la Asociación Americana de Urología, encontrando que sólo el 3.9\% de sus miembros tenía entrenamiento formal en investigación. ${ }^{4}$ Según nuestros hallazgos, desde las ultimas dos décadas el número de investigaciones médicas se ha incrementado de forma importante, principalmente desde 2009. La producción literaria no tuvo variación significativa desde 1955 hasta el año 1999, donde pueden haber influido dos hechos importantes: el primero es la aparición del término medicina basada en la evidencia, acuñado hace 29 años por Gordon Guyatt en la Universidad McMaster, y el segundo, es el nacimiento de Cochrane en 1993, lo que pudo haber impulsado el aumento progresivo a partir de esos años ${ }^{12,13}$

Así mismo, Roth y Siemens encontraron que sólo la mitad de los jefes de residentes de urología consideraban haber tenido educación formal en medicina basada en la evidencia, ${ }^{14}$ y otro estudio realizado por Scales et al, ${ }^{7}$ demostró que los trabajos de investigación en urología frecuentemente tienen errores de diseño y análisis estadístico, lo cual podría mejorar si durante la formación urológica se profundiza en la enseñanza en investigación, mejorando así la calidad de la producción intelectual en esta área. El marcado incremento de publicaciones en la ultima década puede ser influenciado por la adhesión de los principios de la medicina basada en la evidencia a una reforma de salud en la legislación de los Estados Unidos, que se realizí en el 2010, lo que fue también duplicado por la política de salud del Reino Unido. ${ }^{15}$

En cuanto a las revistas con mayor porcentaje de publicaciones en el tema, encontramos que la mayoría de artículos se hallan en aquellas con bajo factor de impacto. 

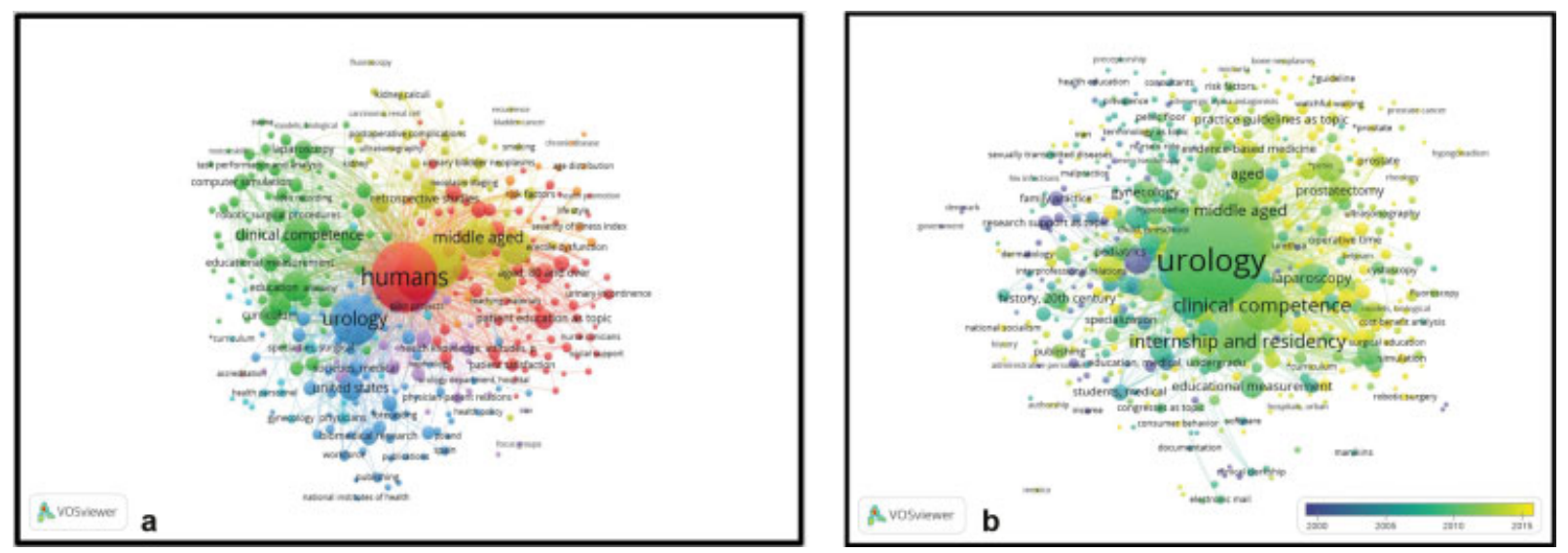

Fig. 5 (a) Análisis de co-ocurrencia de palabras clave en los estudios de educación en investigación en urología. 6a Mapeo y agrupamiento de las palabras claves. (b) Mapeo y agrupamiento de las palabras claves en línea de tiempo entre 2010-2018 (palabras en azul aparecen primero y palabras en amarillo después).

Llama la atención que la revista con mayor factor de impacto en la especialidad (European Urology), no está dentro de las primeras cinco posiciones.

En el análisis de co-ocurrencia realizado a través de VOSViewer, pudimos identificar 3 grupos en el mapeo y análisis de palabras clave: En el primero de ellos predomina la palabra humanos, lo cual es esperable, ya que el fin último de la investigación es mejorar la calidad de vida de los pacientes y es un tópico reiterativo en las publicaciones aunque este no sea el interés principal de las mismas o su objetivo. Para los demás grupos los términos a resaltar tienen una repetición significativa y está en directa relación con el propósito de la investigación, encontrando así cómo el Grupo 2se basa en la educación a los residentes. En este se describe que dentro del curriculum se deben tener parámetros para la evaluación de conocimientos como medición de habilidades y competencias clínicas y competencias en investigación. Finalmente el Grupo 3 se basa principalmente en el esfuerzo que deben realizar las sociedades científicas y programas académicos en la educación médica continuada, la enseñanza en investigación clínica y la producción intelectual. Es llamativo ver cómo en una línea de tiempo los artículos más recientes de investigación en el campo de urología han tenido interés en los análisis de costo efectividad y la medicina basada en la evidencia, lo que señala el cambio en el pensamiento de los investigadores y la ruta en la que posiblemente se direccionarán en un futuro cercano las próximas publicaciones.

Identificamos algunas limitaciones para el desarrollo del presente estudio: la barrera del idioma, encontrando que la mayoría de las publicaciones son en inglés y que aún sin limitar el idioma en nuestra búsqueda puede existir literatura que no se haya incluido para este estudio. Es

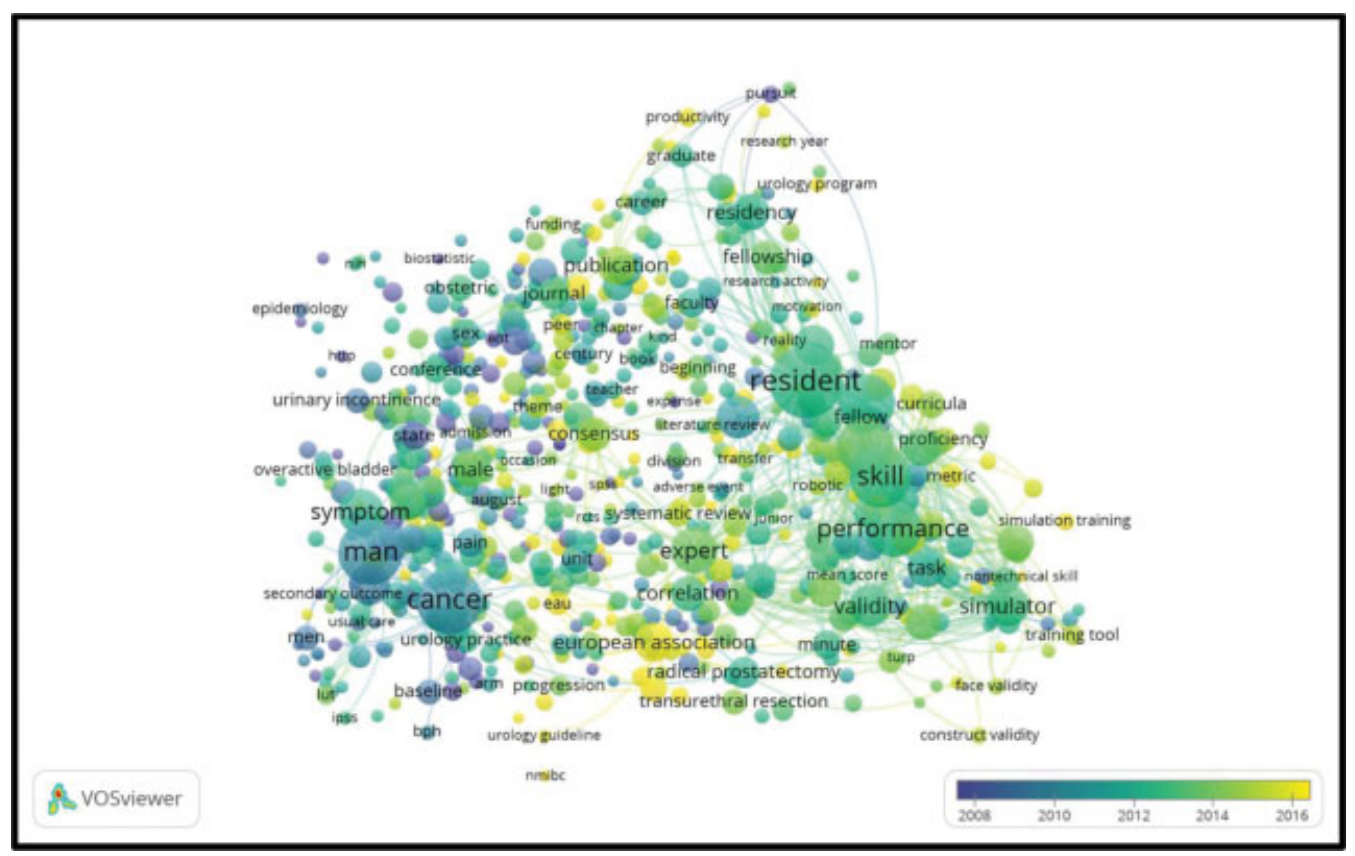

Fig. 6 Mapeo y agrupación de títulos y palabras clave según la frecuencia de aparición entre 2010 y 2018. 
claro que para superar esta barrera las herramientas de búsqueda utilizadas en este trabajo son unas de las más confiables para este tipo de análisis. ${ }^{16,17}$

Debe considerarse que los motores de búsqueda, como PubMed, solo incluyen la dirección del primer autor, por lo que no se puede determinar la cantidad de artículos de colaboración multinacional, y esto a su vez reduciría la posibilidad de tener un cálculo confiable de la producción en países en desarrollo.

Es importante resaltar que la metodología de búsqueda no solo puede reproducirse, sino que ya ha sido utilizada por otros autores, por lo que nuestros resultados pueden ser comparados con futuros manuscritos. Una de las limitaciones es que dentro de la base de datos pueden existir artículos dentro de la búsqueda que cubren otro tipo de tópicos.

A pesar de las limitaciones mencionadas, este es el primer análisis bibliométrico sobre publicaciones relacionadas con la educación en investigación en el campo de la urología. Aunque probablemente no se tuvieron en cuenta la totalidad de los artículos, consideramos que muestra de forma clara el panorama de la publicación en educación sobre investigación en las últimas décadas.

Creemos que este estudio es un instrumento útil para investigadores, pudiendo convertirse en el punto de partida para definir las necesidades de futuras líneas de investigación y trabajos científicos que intenten fortalecer la producción intelectual en urología.

\section{Conclusión}

El estado de la educación en investigación en urología demuestra un claro crecimiento en la producción científica en Estados Unidos, Reino Unido y Turquía, especialmente en la última década; consiguiendo la publicación de la mayoría de los artículos en revistas con bajo factor de impacto, sin encontrar una importante contribución latinoamericana.

Para poder desarrollar una creación intelectual de calidad, se debería invertir en tiempo y recursos para un adecuado entrenamiento en investigación en los programas formación en urología.

Conflicto de intereses

Los autores declaran no tener ningún conflicto de intereses.

\section{Referencias}

1 Acuña cordero ranniery Siete razones por las que vale la pena investigar en medicina en colombia Rev.fac.med [internet]. 2014 dec [cited 2020 mar 08]; 22 (2) : 92-100. Available from:http:// www.scielo.org.co/scielo.php?script=sci_arttext\&pid=s0121$52562014000200010 \& \operatorname{lng}=$ en

2 Roth K, Siemens DR. The status of evidence-based medicine education in urology residency. Can Urol Assoc J 2010;4(02): $114-120$

3 De Battisti F, Salini S. robust analysis of bibliometric data. Stat Methods Appl 2013;22:269-283

4 Dahm P, Poolman RW, Bhandari M, et al; American Urological Association Membership. Perceptions and competence in evidence-based medicine: a survey of the American Urological Association Membership. J Urol 2009;181(02):767-777

5 Scales CD Jr, Voils CI, Fesperman SF, et al. Barriers to the practice of evidence-based urology. J Urol 2008;179(06):2345-2349, discussion 2349-2350

6 Speckman JL, Byrne MM, Gerson J, et al; Consortium to Examine ClinicaL Research Ethics. Determining the costs of institutional review boards. IRB 2007;29(02):7-13

7 Scales CD Jr, Norris RD, Peterson BL, Preminger GM, Dahm P. Clinical research and statistical methods in the urology literature. J Urol 2005;174(4 Pt 1):1374-1379

8 Sweileh WM, Zyoud SH, Al-Jabi SW, Sawalha AF. Assessing urology and nephrology research activity in Arab countries using ISI web of science bibliometric database. BMC Res Notes 2014;7:258

9 Solano C, Rueda Quijano SM, Pinto Briceño NE, Méndez Zaraza AL, Ordoñez Llanes KJ, Tarazona N. El lenguaje de la nefrolitotomía percutánea: un análisis bibliométrico de 32 años de literatura médica. Urología Colombiana 2017;26(03):214-218

10 Rosenkrantz AB, Chung R, Duszak R Jr. Uncited Research Articles in Popular United States General Radiology Journals. Acad Radiol 2019;26(02):282-285

11 Majzoub A, Al Rumaihi K, Al Ansari A. The world's contribution to the field of urology in 2015: A bibliometric study. Arab J Urol 2016;14(04):241-247

12 Guyat G. Preface. En: guyatt g, rennie d (eds.) User's guide to the medical literature. Essentials of evidenced medicine

13 Eddy DM. Evidence-based medicine: a unified approach. Health Aff (Millwood) 2005;24(01):9-17

14 Roth K, Siemens DR. The status of evidence-based medicine education in urology residency. Can Urol Assoc J 2010;4(02): 114-120

15 Dahm P, Konety BR. Evidence-based medicine in urology. World J Urol 2011;29(03):255-256

16 Falagas ME, Pitsouni EI, Malietzis GA, Pappas G. Comparison of PubMed, Scopus, Web of Science, and Google Scholar: strengths and weaknesses. FASEB J 2008;22(02):338-342

17 Kulkarni AV, Aziz B, Shams I, Busse JW. Comparisons of citations in Web of Science, Scopus, and Google Scholar for articles published in general medical journals. JAMA 2009;302(10):1092-1096 